\title{
The Protocol For The Prehabilitation For Thoracic Surgery Study: A Randomized Pragmatic Trial Comparing A Short Home-Based Multimodal Program To Aerobic Training In Patients Undergoing Video-Assisted Thoracoscopic Surgery Lobectomy
}

\section{Yuchao Liu}

Chinese Academy of Medical Sciences \& Peking Union Medical College Hospital Department of anesthesiology https://orcid.org/0000-0003-1809-0668

Zijia Liu ( $\square$ liu-zj02@126.com )

https://orcid.org/0000-0002-1866-4337

\section{Yuelun Zhang}

Chinese Academy of Medical Sciences and Peking Union Medical College Hospital Department of Medical Research Center

\section{Yushang Cui}

Chinese Academy of Medical Sciences \& Peking Union Medical College Hospital Department of Thoracic Surgery

\section{Lijian Pei}

University of the Chinese Academy of Sciences \& Peking Union Medical College Hospital Department of Anesthesiology

\section{Yuguang Huang}

Chinese Academy of Medical Sciences \& Peking Union Medical College Hospital Department of Anesthesiology

\section{Study protocol}

Keywords: Multimodal prehabilitation, aerobic training, video-assisted thoracoscopic surgeries, lobectomy, pragmatic trial, functional capacity, 6-minute walk distance

Posted Date: June 28th, 2021

DOl: https://doi.org/10.21203/rs.3.rs-64845/v1

License: (c) (i) This work is licensed under a Creative Commons Attribution 4.0 International License. Read Full License 


\section{Abstract}

Background: Prehabilitation has been shown to have a positive effect on the postoperative recovery of functional capacity in patients undergoing video-assisted thoracoscopic surgery (VATS) lobectomy. The optimal way to implement prehabilitation programs, such as the optimal forms of prehabilitation, duration, intensity, and methods to improve compliance, remained to be studied. This Prehabilitation for Thoracic Surgery Study will compare the effectiveness of multimodal and aerobic training-only programs in patients undergoing thoracoscopic lobectomy.

Methods: This randomized pragmatic trial will be conducted in Peking Union Medical College Hospital (PUMCH) and include 100 patients who are eligible to undergo VATS lobectomy. Patients will be randomized to a multimodal or aerobic training group. Prehabilitation training guidance will be provided by a multidisciplinary care team. The patients in the multimodal group will perform aerobic exercises, resistance exercises, breathing exercises, psychological improvement strategies, and nutritional supplementation. Meanwhile, the patients in the aerobic group will conduct only aerobic exercises. The interventions will be home-based and supervised by medical providers. The patients will be followed up until 30 days after surgery to investigate whether the multimodal prehabilitation program differs from the aerobic training program in terms of the magnitude of improvement in functional capability pre- to postoperatively. The primary outcome will be the perioperative 6-minute walk distance (6MWD). The secondary outcomes will include the postoperative pulmonary functional recovery status, health-related quality of life score, incidence of postoperative complications, and clinical outcomes.

Discussion: Prehabilitation remains a relatively new approach that is not widely performed by thoracic surgery patients. The existing studies mainly focus on unimodal interventions. While multimodal prehabilitation strategies have been shown to be preferable to unimodal strategies in a few studies, the evidence remains scarce for thoracic surgery patients. The results of this study will contribute to the understanding of methods for thoracoscopic lobectomy patients.

Trial registration: ClinicalTrials.gov, NCT04049942, registered on August 8, 2019, https://clinicaltrials.gov/ct2/show/NCT04049942

\section{Background}

Lung cancer remains the leading cause of cancer-related deaths worldwide [1]. For localized non-small cell lung cancer (NSCLC), surgical resection with radical lymph node dissection is a potentially curative option [2]. Surgical techniques have advanced, and video-assisted thoracoscopic surgery (VATS) is currently a well-established and preferred choice at many institutions due to its minimally invasive nature [3]. Despite advancements in the management of NSCLC patients occurring, functional capacity and pulmonary function in patients undergoing thoracoscopic surgeries are still believed to decrease significantly after surgery, and the incidence of postoperative pulmonary complications (PPCs) is 
reported to be $12 \%-40 \%[4,5]$. These factors impede patient recovery and potentially increase patients' length of hospital stay (LOS).

One of the approaches that has been developed to improve the outcomes of surgical patients is prehabilitation, which refers to a proactive process of optimizing the functional capacity of an individual before surgery [6]. Although the concept of prehabilitation was established two decades ago and has since been prompting many investigational efforts, it remains, to this day, a work in progress, especially in the thoracic surgery community.

Prehabilitation is most extensively investigated in the gastrointestinal cancer community. A wide range of prehabilitation strategies that have been conducted in patients undergoing gastrointestinal surgeries, including exercise-based interventions, nutritional optimization, psychological optimization, smoking and/or alcohol cessation, in the form of unimodal or multimodal programs, have improved functional capacity [6-10]. Several small studies have shown results that favor multimodal strategies over unimodal strategies $[8,11]$, although conclusions have yet to be drawn about the most feasible and achievable manner of prehabilitation.

The necessity of prehabilitation is widely recognized in the thoracic surgery community because thoracic surgery is associated with functional impairments and a relatively high incidence of PPCs [12]. Until recently, most of the preoperative interventions that have been implemented in thoracic surgery patients have been unimodal, and the majority of previous studies have focused on exercise-based training programs, particularly aerobic exercises [13-15]. Although there is increasing evidence that preoperative exercise training improves physical function and pulmonary function, it remains unclear how exercise training compares to other forms of prehabilitation in efficacy and whether improvements in functional capacity lead to better postoperative outcomes.

Our team conducted a randomized controlled trial in 2017 (registration number NCT03068507) to examine the efficacy of a 2-week multimodal home-based prehabilitation strategy in patients undergoing thoracoscopic surgeries for lung cancer. The multimodal program included physical exercise, respiratory training, nutritional supplements, and psychological optimization. This study yielded promising results, showing that a short-term home-based multimodal prehabilitation program could improve perioperative functional capacity in patients undergoing thoracoscopic lobectomy, as indicated by an improved perioperative 6-minute walk distance (6MWD) [16].

The Prehabilitation for Thoracic Surgery Study aims to further understand the role of prehabilitation in thoracoscopic surgery patients by comparing the efficacy of a short multimodal home-based prehabilitation program with an aerobic exercise-only prehabilitation program, as the latter has been most extensively investigated to date.

\section{Research question}


The aim of the Prehabilitation for Thoracic Surgery Study is to answer the following question: is shortterm multimodal prehabilitation more beneficial than aerobic exercise-based prehabilitation in improving perioperative functional walking capacity in patients undergoing VATS lobectomy?

\section{Methods}

\section{Trial design}

The Prehabilitation for Thoracic Surgery Study is a randomized pragmatic trial comparing the impact of a short home-based multimodal prehabilitation program to a preoperative aerobic training program in patients undergoing VATS for lung cancer. The overall trial design is illustrated in Fig. 1 (see Additional file 1 for the standard protocol items: recommendations for interventional trials (SPIRIT) checklist). Ethics board approval of this study was obtained from Peking Union Medical College Hospital (PUMCH) Institutional Review Board on July 23, 2019. This study will be conducted in PUMCH by a multidisciplinary care team, including anesthesiologists, thoracic surgeons, nutritionists and rehabilitation medicine practitioners. After they are recruited and provide consent, the patients will be randomized to two study arms; then, they will be instructed to undergo either a tailored aerobic program or a multimodal prehabilitation program during the period of time prior to surgery. The patients will be followed up until 30 days after surgery. The effects of the two arms will be assessed on the basis of physical capacity test results (6MWD), pulmonary function test results, patient-reported qualitative outcomes, clinical outcomes and perioperative laboratory results. The patients, care providers and researchers in charge of enrolling, following up, and assessing outcomes (performing the 6MWT) will not be blinded to allocation due to the nature of the intervention. Data analysts will be blinded. Generally data analysts will not need to be unblinded.

\section{Participant eligibility criteria}

The participating patients must be (1) thoracic surgery outpatients in $\mathrm{PUMCH}$; (2) aged from 18 years to 70 years; (3) suspected of having lung cancer; and (4) scheduled for elective thoracoscopic surgery in PUMCH. Patients will be excluded from the study if they (1) decline to participate in the study (due to any reason); (2) have an American Society of Anesthesiologists (ASA) grade > III; (3) plan to undergo or have undergone neoadjuvant therapy; (4) are unable to tolerate any component of the multimodal prehabilitation or aerobic program (including exercises, whey protein supplements, and functional capacity tests); or (5) have other severe cardiopulmonary diseases that may affect the 6MWD results.

\section{Patient recruitment and allocation}

In PUMCH, approximately 100-150 surgeries are scheduled each month. Patients will be recruited from the thoracic surgery outpatient department. When they are scheduled for VATS lobectomy, they will be screened for eligibility and asked to provide informed consent by a research staff. The patients included will then be randomly assigned to two equally-sized groups, the multimodal prehabilitation group and the aerobic prehabilitation group, on the basis of a computer-generated sequence. The random allocation results will be concealed using sealed opaque envelopes. One of the main researchers of the study 
designs the statistical analysis plans and will be generating the allocation sequence. Two experienced anesthesiologist researchers will be in charge of enrolling and assigning participants according to the randomization result.

\section{Baseline interview}

Upon patient recruitment and allocation to groups, a one-on-one baseline interview and evaluation will be conducted to (1) record patients' comprehensive medical history; (2) assess patients' nutritional states; (3) assess patients' functional capacity during the 6-minute walk test (6MWT); (4) conduct a pulmonary function test (Spirodoc portable pulmonary function meter, Medical International Research S.r.l., 09000261972), during which the forced expiratory volume in one second (FEV1, L), FEV1\%, forced vital capacity (FVC, L), FVC\%, FEV1/FVC, peak expiratory flow (PEF, L/s), and PEF\% will be recorded; (5) record laboratory results, if applicable, including the levels of alanine transaminase (ALT), creatine (CR), Creactive protein (CRP), albumin (Alb), glucose (Glu), and myocardial enzymes; (6) evaluate the patients' baseline quality of life and mental state using standard tools [hospital anxiety and depression scale (HADS), World Health Organization disability assessment schedule II (WHODAS 2.0) score, postoperative quality of recovery score - 15 (QoR-15)]; (7) explain in detail the prehabilitation plan for both groups; (8) encourage patients to choose a preferred way to be followed up, such as by telephone, short text messages or WeChat messaging.

\section{Interventions}

Aside from the prehabilitation strategies, all patients enrolled will receive conventional perioperative recommendations, including those based on preoperative anesthesia assessments, drug treatment recommendations for chronic diseases, and recommendations to quit smoking and practice abstinence. The patients will also be provided information on anesthesia and surgical processes, according to each individual's needs.

We will encourage the patients in both groups to choose their preferred types of exercise and form individual-specific prehabilitation plans. A prehabilitation instruction brochure will be distributed to patients along with a daily self-evaluation prehabilitation journal, where they can record their adherence to the prehabilitation plans. Standardized short message interviews will be sent to patients twice a week in the manner chosen by the patients to optimize adherence and promote timely feedback. The patients will receive intraoperative and perioperative care according to standard practice in terms of the surgical procedures, anesthesia and analgesia management and nursing care.

The patients will be encouraged to reach out to our researchers if there are injuries or discomforts during the follow-up period. Since intervention is based on moderate exercise and nutrition supplement, and exercise plan is individualized to consider participants' capacity and preference, harm of the intervention is considered minimal. However, if patients experience injuries or discomforts due to the exercises or whey protein supplement, the intervention will be discontinued and we plan to offer appropriate medical and financial support. At any point of the study period, if patients request to stop or pause with the intervention, the intervention will be discontinued. 
For the multimodal group, we will implement the following prehabilitation strategy:

1) A 30-minute aerobic exercise session will be performed at least 3 times a week; it will be divided into a 5-minute warm-up (including ankle extensor lifts and static quadriceps contraction) and a 25-minute aerobic exercise in the form of jogging, power walking or cycling, depending on the patients' individual choice. We will encourage patients to tailor their exercise intensity to the Borg rating of perceived exertion (RPE) scale [17] shown by Table 1., and target heart rate (HR), increasing or decreasing the intensity according to the level of exertion throughout the prehabilitation period. We will recommend that patients achieve moderate to high levels of exertion, as indicated by 13-16 points on the Borg RPE scale and a target HR of (220-age-resting HR) $\times 70 \%+$ resting HR. The methods of using the Borg RPE scale and target HR to adjust the exercise intensity will be explained at the baseline interview, and both methods will be illustrated in the brochure we give the patients. We will also provide each patient with a pulse oximeter (Jiangsu Yuyue Medical Equipment \& Supply Co., Ltd., 20172201070) to help monitor HR. For patients who choose an outdoor activity as their aerobic exercise training method, we will stress the importance of safety and advise them to avoid exercising outdoors on days with poor air quality index values $(>100)$.

Table 1. Borg RPE scale 


\begin{tabular}{|ll|}
\hline Score & Level of exertion \\
\hline 6 & No exertion at all \\
\hline 8 & Extremely light \\
\hline 9 & Minimal recognition of effort \\
\hline 10 & Could just start to beware of breathing \\
\hline 11 & Fairly light (conversation is easy and feel like you could keep the pace for a long time) \\
\hline 12 & Light \\
\hline 13 & Somewhat hard, but still feel able to continue \\
\hline 14 & You could hear your breathing clearly but not struggling \\
\hline 16 & Hard (You could talk but not in full sentences) \\
\hline 17 & Very hard. You could continue but would be pushing yourself to feel very fatigued \\
\hline 18 & You could not talk because of the heavy panting \\
\hline 19 & Extremely hard \\
\hline
\end{tabular}

2) Guided resistance exercise will be performed at least twice a week. At the baseline interview, a research staff qualified in performing physical therapy training and evaluations will initially evaluate the physical status of the patients and then explain and demonstrate the resistance exercises. We will provide each patient in the multimodal group with two pulling straps of different elasticities. Resistance training will be performed with either strap, depending on the patient's individual strength limits. Most patients will start training with the less elastic strap and advance to the more elastic strap once they feel that training with the less elastic strap requires too little effort. We will assign 4 pre-set resistance postures for the training sessions, involving 4 major muscle groups (upper and lower limbs, chest and core muscles). The complete resistance training session will include 3 sets of exercises with an interval of 2 minutes between each set. Each set will include 10-12 repetitions of the 4 resistance postures. At the end of each complete session, the patients will be asked to evaluate their perceived exertion by using the Borg RPE scale. The target score will be 13-16. The number of repetitions performed within each set and the strap selection should be adjusted on an individual basis throughout the prehabilitation period according to the Borg RPE scores.

3) Breathing exercises will be performed at least 3 times per day, with each session lasting more than 10 minutes. We will introduce three types of exercises: (1) guided effective coughing (sit up and lean slightly forward, inhale fully then cough out in a short and forceful manner while engaging core muscles), (2) 
blowing up a small balloon in one breath and blowing it up slowly for more than 5 seconds, (3) breathing training with the Tri-Ball Respiratory Exerciser (Leventon S.A., Barcelona, Spain) that we will distribute. All three types of breathing exercises will be demonstrated at the baseline interview and explained in the brochure. The patients will be allowed choose to conduct one or more types of breathing exercises in one session.

4) Regarding nutritional advice and whey protein supplementation, patients' nutritional status and dietary habits will be evaluated at baseline by a research staff member trained in nutriology. Nutritional advice predetermined by our nutritionists will be given to modulate the patient's eating habits, mainly aiming at reducing fat-rich diets and increasing high-quality protein uptake. Patients with malnutrition will be advised to increase their calorie uptake. We will also provide patients in the multimodal group with whey protein powder (Inerish; Sino-American Medical Institute Inc, San Diego, CA) as a protein supplement and advise them to take it daily ( $20 \mathrm{~g} /$ day for males and $15 \mathrm{~g} /$ day for females). If patients plan on performing exercises that day, we will advise that the whey protein powder is taken within $1 \mathrm{~h}$ postexercise, promoting muscle synthesis.

5) Psychological therapy will include listening to soothing music, broadcasts or other relaxing activities of the patients' choice before sleep. We will provide each patient in the multimodal group with a simple music player with prerecorded relaxing, classical music tracks and encourage patients to use it if they prefer this type of relaxation method. We will also encourage patients to choose their preferred methods of relaxation, including listening to audiobooks, meditating, watching relaxing television shows, etc.

\section{Aerobic prehabilitation strategy features}

The aerobic group will receive the same instructions regarding the individualized home-based aerobic exercise program as the multimodal group will, but they will not receive advice regarding the other aspects of their behaviors. These patients will also be advised to record their daily exercises in a prehabilitation journal and will receive the same follow-up interviews twice a week.

\section{Outcomes}

The primary outcome will be the perioperative 6MWD (baseline, 1 day presurgery and 30 days postsurgery). The 6MWT will be conducted following the guidelines of the American Thoracic Society (ATS) [18]. The research staff members who will be involved in performing the 6MWT will all be trained before administering this test. We will conduct the 6MWT indoors in a 30-meter-long corridor in PUMCH. We will ensure that supplies needed for safety issues are readily available whenever the 6MWT is performed, including oxygen, sublingual nitroglycerine, aspirin, and albuterol. All the staff members who will be involved have been previously certified in cardiopulmonary resuscitation. The 6MWT will be conducted in a standard manner. From the 10-minute rest period before the test to all the verbal instructions provided to the patients before and during the test, all procedures will be performed in accordance with the standard ATS guidelines.

We will record patients' sex, age, height, weight, medications before the test, and whether they need oxygen supplementation during the test. We will measure blood pressure, HR, oxygen saturation, and the 
Borg RPE score before and after the test. We will note the number of laps and calculate the 6MWD.

The secondary outcomes will include the perioperative pulmonary function test results, including the FEV1, FEV1\%, FVC, FVC\%, FEV1/FVC, PEF, and PEF\% values (baseline, 1 day presurgery and 30 days postsurgery); the perioperative HADS and WHODAS 2.0 (baseline, 1 day presurgery and 30 days postsurgery); post-surgery QoR-15 (3 consecutive days after surgery); prognostic information (mortality and morbidity, length of hospital stay, ICU stay time, duration of chest tube placement duration); and perioperative laboratory results, if applicable, including the levels of ALT, CR, CRP, Alb, Glu and myocardial enzymes (baseline, 1 day presurgery and 30 days postsurgery).

\section{Implementation timeline}

The timeline of this study is 6 to 8 weeks; prehabilitation will be implemented for 2 to 4 weeks before surgery, and the follow-up period will include the 4 weeks after surgery. The timeline will differ across individuals, mainly due to the wait time before surgery. Clinical outcome data will be retrieved by practitioners from the inpatient records within one month after surgery. Figure 2 shows the full timeline.

\section{Sample size}

In this study, we will calculate the sample size on the basis of a comparison of the measured primary outcome, the 6MWT result, between a small pilot study and a previous multimodal prehabilitation study conducted in our institute [16]. We will assume the average 6WMD in the aerobic prehabilitation group to be $30 \mathrm{~m}$ shorter than that in the multimodal group at 30 days postoperatively. The standard deviation (SD) for the 6MWD will be estimated to be 50 meters for both groups. A sample size of 45 patients each in the aerobic group and multimodal prehabilitation group is required to detect a statistically significant difference at a two-sided significance level of 0.05 and statistical power of $90 \%$. To account for patient dropout and missing data, we plan to recruit a total of 50 patients for each group.

\section{Data collection}

Upon receiving informed consent, the researchers will collect the patients' medical history and demographic information at the baseline interview. Baseline laboratory test results will then be retrieved from patients' electronic medical records: complete blood count (CBC), liver and kidney function test results, electrolyte levels, and coagulation routine test results.

The 6MWD and pulmonary function test will be conducted and recorded by trained researchers at our institution after they provide standardized instructions and demonstrations of the tests. Patient-reported qualitative questionnaires will be administered by and the results will be recorded by trained researchers.

During the prehabilitation period, message interviews will be sent to patients twice a week by our researchers, and feedback on weekly prehabilitation performance will be recorded.

After surgery, designated clinicians will retrieve the surgical approach performed, tumor assessment results, pathology results and postoperative complications from the patients' electronic records. 
All the data will be collected by researchers who have signed a confidential disclosure agreement. Patients' identity and personal information will not be disclosed without consent.

All data collected will be recorded first in the paper-based case report form (CRF) and then entered into a digital database by an independent investigator. The digital database will be under the supervision by the Data Monitoring Committee (DMC) composed of two experienced doctors of department of anesthesiology, and one statistician of department of medical research center, $\mathrm{PUMCH}$, who are independent from this study. The DMC members will monitor the integrity of the data and patient safety. Upon data entry, the database will be locked by a password. DMC members will make the decision to unblind, conduct an interim analysis or terminate the trial, if they deem it necessary.

\section{Statistical Analysis}

Patients' baseline characteristics will be described by descriptive analysis and compared between groups to detect clinically relevant differences. If evident baseline imbalance exists, the analysis of the primary outcome will be adjusted. For tests conducted perioperatively including 6MWD, pulmonary function test results, HADS and WHODAS 2.0 score, a linear mixed-effects model will be built to analyze the interaction between intervention and time [19]. Other secondary outcomes including the QoR-15 score, LOS, chest tube duration, postoperative complications will be analyzed using $\chi 2$ test and the Mann-Whitney $U$ where appropriate. Patients lost to follow-up will be considered as non-adherence. We will perform sensitivity analysis to test if the analyses of the primary outcome will be robust to the missing values. All statistical analyses will be completed in R (R Foundation for Statistical Computing, Vienna, Austria; version 3.5.2).

\section{Dissemination plans}

We plan to communicate trial results thoroughly with our multidisciplinary team and encourage conversations with medical providers in other state facilities during annual conference of Chinese Medical Association. These medical providers will in turn disseminate results to the patient community. We will also inform the patient community of the results during pre-surgery evaluation and consults. We are planning to invite researchers from our multidisciplinary team as co-authors in the publication process, and we are not planning to use professional writers. For further dissemination of the results, we will post the study concept and major results on the website of the anesthesiology department of PUMCH. We will be open to provide the whole study protocol as well as patient-level dataset when approached by interested health providers or researchers.

\section{Trial Status}

This manuscript is based on version 2.1 of our study protocol, updated July 22, 2020. Enrollment started on November 9, 2019, then went on pause during January 2020 due to the COVID-19 pandemic. Enrollment restarted gradually in July 2020. Completion of recruitment is expected to be November 2021. If there will be important modifications to protocols related to enrolment, prehabilitation strategies, outcomes and statistics plans, we will inform all researchers and DMC members, report to our institutional Institutional Review Board and make timely updates on ClinicalTrials.gov and related journal. 


\section{Discussion}

The Prehabilitation in Thoracic Surgery Study investigates two types of prehabilitation strategies in patients undergoing VATS, a potentially more promising multimodal strategy and a more recognized aerobic exercise strategy, to answer the question of whether the former is more beneficial than the latter.

As gastrointestinal cancer is the most extensively explored condition regarding prehabilitation, there is a large amount of evidence showing that a multimodal strategy is more beneficial than a unimodal strategy in improving functional capacity recovery postsurgery. Our group formerly conducted a trial demonstrating that a short-term home-based multimodal prehabilitation strategy improves postsurgical 6MWD, a well-established marker for functional capacity. Most of the studies on prehabilitation in VATS patients included unimodal strategies, and aerobic exercise is widely considered to be the most important factor. We hope this study will shed more light on the differences in prehabilitation strategies in VATS patients.

This study is a pragmatic trial, and we made an effort to create a feasible and flexible protocol tailored to patients' individual needs and preferences to promote adherence to training strategies and achieve viable, effective prehabilitation outcomes. Patient interviews make up a crucial part of the study, as they ensure patient safety and the fidelity of protocol adherence data and allow valuable feedback to be collected.

The training protocols for both arms were refined based on feedback from patients and multidisciplinary investigators during our previous study, so the strategy is more patient-centered and efficient. We propose a mixed methods evaluation panel to further assess the efficacy and feasibility of a multimodal prehabilitation strategy compared to aerobic training alone within a thoracoscopic surgery setting. We hope this study will provide more evidence on the prehabilitation methods within the thoracic surgery community and help promote better and faster functional recovery.

\section{Abbreviations}

VATS: video-assisted thoracoscopic surgery; PUMCH: Peking Union Medical College Hospital; 6MWD: 6minute walk distance; NSCLC: non-small cell lung cancer; PPCs: postoperative pulmonary complications; LOS: length of hospital stay; ASA: American Society of Anesthesiologists; 6MWT: 6-minute walk test; FEV1: forced expiratory volume in one second; FVC: forced vital capacity; PEF: peak expiratory flow; ALT: alanine transaminase; CR: creatine; CRP: c-reactive protein; Alb: albumin; Glu: glucose; HADS: hospital anxiety and depression scale; WHODAS 2.0: World Health Organization disability assessment schedule II; QoR-15: postoperative quality of recovery score -15; RPE: rating of perceived exertion; HR: heart rate; ATS: American Thoracic Society; SD: standard deviation; CBC: complete blood count; CRF: case report form; DMC: Data Monitoring Committee

\section{Declarations}


Ethics board approval of this study was obtained from Peking Union Medical College Hospital (PUMCH) Institutional Review Board on July 23, 2019. Written informed consent will be sought from the participants before the start of the recruitment.

\section{Consent for publication}

Not Applicable

\section{Availability of data and materials}

Not Applicable

\section{Competing interests}

The authors declare that they have no competing interests.

\section{Funding}

This study is supported by the Non-profit Central Research Institute Fund of Chinese Academy of Medical Sciences. The sponsor will not be involved in study design; collection, management, analysis, or interpretation of data; writing of the report; or the decision to submit the report for publication.

\section{Authors' contributions}

$Y L$ contributed in registering the trial and writing the manuscript. $Z L$ designed the study protocol and applied for ethical approval. YZ designed the statistical analysis plans. YC and LP provided inputs on study implementation. YH helped with the revision of the manuscript. All authors read and approved the final manuscript.

\section{Acknowledgements}

Not Applicable

\section{Authors' information}

${ }^{1}$ Department of Anesthesiology, Chinese Academy of Medical Sciences \& Peking Union Medical College Hospital, Beijing 100730, China

2 Department of Medical Research Center, Chinese Academy of Medical Sciences \& Peking Union Medical College Hospital, Beijing 100730, China

${ }^{3}$ Department of Thoracic Surgery, Chinese Academy of Medical Sciences \& Peking Union Medical College Hospital, Beijing 100730, China

\section{References}


1. Huo J, Xu Y, Sheu T, Volk RJ, Shih YT. Complication rates and downstream medical costs associated with invasive diagnostic procedures for lung abnormalities in the community setting. JAMA Intern Med. 2019;179:324-32.

2. Sanchez-Lorente D, Navarro-Ripoll R, Guzman R, Moises J, Gimeno E, Boada M, et al. Prehabilitation in thoracic surgery. J Thorac Dis. 2018;10:2593-600.

3. Berfield KS, Farjah F, Mulligan MS. Video-assisted thoracoscopic lobectomy for lung cancer. Ann Thorac Surg. 2019;107:603-9.

4. Yang R, Wu Y, Yao L, Xu J, Zhang S, Du C, et al. Risk factors of postoperative pulmonary complications after minimally invasive anatomic resection for lung cancer. Ther Clin Risk Manag. 2019;15:223-31.

5. Bertani A, Ferrari P, Terzo D, Russo E, Burgio G, De Monte L, et al. A comprehensive protocol for physiokinesis therapy and enhanced recovery after surgery in patients undergoing video-assisted thoracoscopic surgery lobectomy. J Thorac Dis. 2018;10:499-511.

6. Bolshinsky V, Li MH, Ismail H, Burbury K, Riedel B, Heriot A. Multimodal prehabilitation programs as a bundle of care in gastrointestinal cancer surgery: a systematic review. Dis Colon Rectum. 2018;61:124-38.

7. Boereboom C, Doleman B, Lund JN, Williams JP. Systematic review of pre-operative exercise in colorectal cancer patients. Tech Coloproctol. 2016;20:81-9.

8. Li C, Carli F, Lee L, Charlebois P, Stein B, Liberman AS, et al. Impact of a trimodal prehabilitation program on functional recovery after colorectal cancer surgery: a pilot study. Surg Endosc. 2013;27:1072-82.

9. Levett DZ, Grocott MP. Cardiopulmonary exercise testing, pre- habilitation, and enhanced recovery after surgery (ERAS). Can J Anaesth. 2015;62:131-42.

10. Minnella EM, Awasthi R, Loiselle SE, Agnihotram RV, Ferri LE, Carli F. Effect of exercise and nutrition prehabilitation on functional capacity in esophagogastric cancer surgery: a randomized clinical trial. JAMA Surg. 2018;153:1081-89.

11. Gillis C, Li C, Lee L, Awasthi R, Augustin B, Gamsa A, et al. Prehabilitation versus rehabilitation: a randomized control trial in patients undergoing colorectal resection for cancer. Anesthesiology. 2014;121:937-47.

12. Kaufmann KB, Loop T, Heinrich S. Risk factors for post-operative pulmonary complications in lung cancer patients after video-assisted thoracoscopic lung resection: Results of the German Thorax Registry. Acta Anaesthesiol Scand. 2019;63:1009-18.

13. Cavalheri V, Granger C. Preoperative exercise training for patients with non-small cell lung cancer. Cochrane Database Syst Rev. 2017;6:CD012020.

14. Garcia RS, Yanez-Brage MI, Moolhuyzen EG, Riobo MS, Paz AL, Mate JMB. Preoperative exercise training prevents functional decline after lung resection surgery: a randomized, single-blind controlled trial. Clin Rehabil. 2017;31:1057-67. 
15. Batchelor TJP, Rasburn NJ, Abdelnour-Berchtold E, Brunelli A, Cerfolio RJ, Gonzalez M, et al. Guidelines for enhanced recovery after lung surgery: recommendations of the Enhanced Recovery After Surgery (ERAS(R)) Society and the European Society of Thoracic Surgeons (ESTS). Eur J Cardiothorac Surg. 2019;55:91-115.

16. Liu Z, Qiu T, Pei L, Zhang Y, Xu L, Cui Y, et al. Two-week multimodal prehabilitation program improves perioperative functional capability in patients undergoing thoracoscopic lobectomy for lung cancer: a randomized controlled trial. Anesth Analg. 2019. doi:10.1213/ANE.0000000000004342.

17. Williams N. The borg rating of perveived exertion (RPE) scale. Occup Med. 2017;67:404-5.

18. ATS Committee on Proficiency Standards for Clinical Pulmonary Function Laboratories. ATS statement: guidelines for the six-minute walk test. Am J Respir Crit Care Med. 2002;166:111-7.

19. Schober P, Vetter TR. Repeated measures designs and analysis of longitudinal data: if at first you do not succeed- try, try again. Anesth Analg. 2018;127:569-75.

\section{Figures}




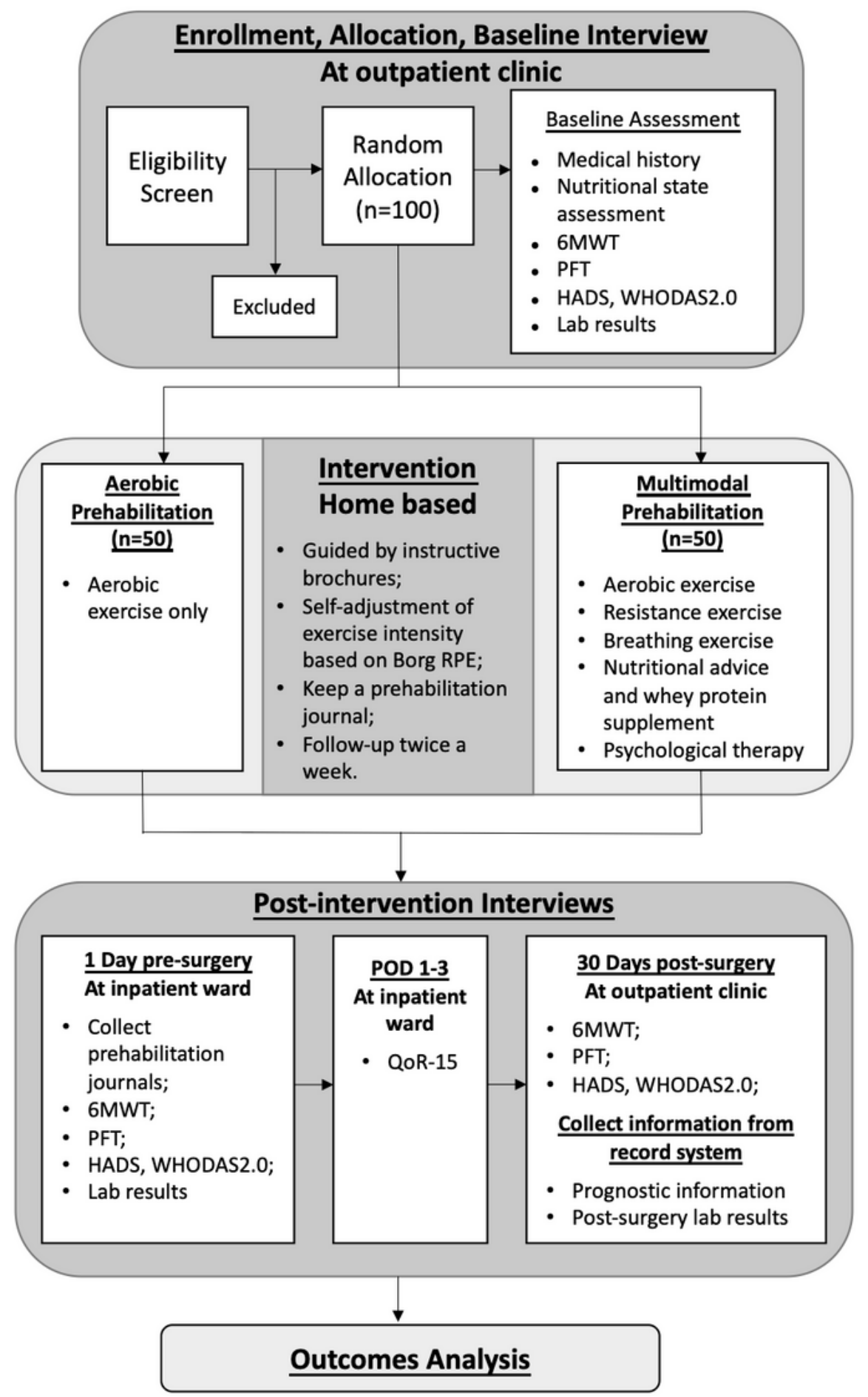

\section{Figure 1}

Flow diagram of the study. 6MWT, 6-minute walk test; PFT, pulmonary function test; HADS, hospital anxiety and depression scale; WHODAS 2.0, World Health Organization disability assessment schedule II; Borg RPE, Borg rating of perceived exertion scale; POD, postoperative day; QoR-15, quality of recovery score -15 . 


\begin{tabular}{|c|c|c|c|c|c|}
\hline & \multicolumn{5}{|c|}{ STUDY PERIOD } \\
\hline & Enrolment & Allocation & intervention & Pre-surgery & Close-out \\
\hline TIMEPOINT & -1 & 0 & 1 & 2 & 3 \\
\hline \multicolumn{6}{|l|}{ ENROLMENT: } \\
\hline Eligibility Screen & $x$ & & & & \\
\hline Informed Consent & $\mathrm{x}$ & & & & \\
\hline Demographics & $\mathrm{x}$ & & & & \\
\hline Medical History & $\mathrm{x}$ & & & & \\
\hline Nutritional Status & $\mathrm{x}$ & & & & \\
\hline Allocation & & $x$ & & & \\
\hline \multicolumn{6}{|l|}{ INTERVENTIONS: } \\
\hline \multirow[t]{2}{*}{ Multimodal Prehablitation } & & & $\mathrm{x}$ & & \\
\hline & & & $\mathrm{x}$ & & \\
\hline \multicolumn{6}{|l|}{ ASSESSMENTS: } \\
\hline 6MWD & $\mathrm{x}$ & & & $\mathrm{x}$ & $\mathrm{x}$ \\
\hline Pulmonary function test & $x$ & & & $x$ & $x$ \\
\hline Qualitative questionnaires & $x$ & & & $x$ & $x$ \\
\hline Laboratory test & $x$ & & $x$ & $x$ & $x$ \\
\hline Compliance & & & $x$ & $x$ & \\
\hline Surgical records & & & & & $x$ \\
\hline Pathology report & & & & & $x$ \\
\hline Clinical outcomes & & & & & $x$ \\
\hline
\end{tabular}

\section{Figure 2}

Timeline according to the SPIRIT guidelines. Timepoints: -1 , enrollment at thoracic outpatient clinic; 0 , allocation; 1 , waiting period before surgery; 2 , day before surgery; 3,30 days after surgery. The pulmonary function test results will include the FEV1, FEV1\%, FVC, FVC\%, FEV1/FVC, PEF, and PEF\% values; the qualitative questionnaires include the HADS, World Health Organization disability assessment schedule II (WHODAS 2.0), and postoperative quality of recovery -15 (QoR-15); the laboratory test results include the 
levels of ALT, CR, CRP, Alb, Glu and myocardial enzymes; the clinical outcomes include the mortality and morbidity, length of hospital stay, ICU stay time, and duration of chest tube placement duration.

\section{Supplementary Files}

This is a list of supplementary files associated with this preprint. Click to download.

- SPIRITChecklist2.doc 